lower levels of ACP in people from Black, Asian and Minority Ethnic (BAME) communities. BAME groups are also more likely to desire invasive medical interventions, regardless of prognosis and impact on quality of life. Little is known about how the model of resuscitation decision-making fits with the social, cultural and religious values and beliefs of BAME groups. Evidence also suggests that health care professionals (HCPs) report a lack of confidence in having culturally appropriate discussions with BAME patients and their families. Equipping professionals to be more confident about such ACP discussions with BAME patients may lead to achieving patient preferences.

Aims With a focus on making decisions about resuscitation, this study explores professional views and experiences of ACP with patients from BAME backgrounds. It aims to identify barriers and enablers and person-centred outcomes to such discussions and provide evidence for training professionals.

Methods Thematic analysis of qualitative semi-structured interviews with HCPs across primary, secondary and tertiary care in Leicester, including GPs, hospital doctors and nurses.

Results There was an emphasis on the significance of building rapport, the timing of discussions and navigating communication barriers. Barriers to decision-making included: patients' and their families understanding of both prognosis and resuscitation; and differing values amongst generations of migrants. Professionals struggled with how to find a balance between acting in a non-discriminatory way whilst respecting cultural differences. Most HCPs highlight the need for further training and/or support.

Conclusion There are significant barriers for HCPs when discussing resuscitation decisions with people from BAME communities. This increases the complexity of navigating ACP and achieving patients' preferences. HCPs would benefit from further training and support.

\section{P-16 CONVERSATIONS ON LIVING AND DYING: FACILITATING ADVANCE CARE PLANNING FOR OLDER PEOPLE WITH FRAILTY}

${ }^{1}$ Sarah Combes, ${ }^{1,2}$ Caroline Nicholson, ${ }^{1}$ Karen Gillett, ${ }^{1}$ Christine Norton. ${ }^{1}$ King's College London, London, UK; ${ }^{2}$ St Christopher's Hospice, London, UK

\subsection{6/bmispcare-2017-hospice.43}

Background Older people living with frailty (older people) often receive suboptimal end-of-life care (Lloyd et al., 2016). Frequently under- or over-treated, older people experience inappropriate hospital admissions and procedures (Houben et al., 2014; Hunt et al., 2014). Most die in hospital despite $81 \%$ wishing to die at home (Office for National Statistics 2016). Advance care planning (ACP), a structured conversation between professionals and someone nearing the end-of-life, enables people to discuss and document what matters to them regarding their future care (Thomas \& Lobo, 2011) Facilitating informed decision-making regarding ACP means people are more likely to receive person-centred end-of-life care (National Council for Palliative Care, 2011). Multiple challenges mean that ACP is relatively uncommon for older people (Musa et al., 2015; Pollock \& Wilson, 2015). Consequently, priorities are often not discussed prior to significant deterioration (Sharp et al., 2013). leading to crisis decision-making which older people may not have capacity for (Clegg, 2013). Current initiatives encompass aspects of ACP.
However, there is no systematic, agreed format or training for health and social care professionals (professionals) that supports older people to articulate their broader end-of-life wishes on an ongoing basis (Brinkman-Stoppelenburg et al., 2014; Johnson et al., 2015; Weathers et al., 2016).

Aims This four-phase study aims to develop a relevant ACP intervention in collaboration with older people, their carers and professionals. Phase one, presented here, aims to establish current evidence regarding the components and implementation of ACP for community-dwelling older people, and use this to develop a theoretical model which will underpin the remaining study phases.

Methods An integrative review will be conducted using CINAHL, Embase, Ovid Medline, PsycINFO, and NHS Evidence databases, grey literature, and hand-searching. Paper selection will be verified by the research team and critically appraised using relevant systematic tools. Whittemore and Knafl's (2005) method will be used to develop an integrated, theoretical model. This will include data mapping against COM-B, a model of behaviour change often used in intervention development, to identify relevant target behaviours. (Michie et al., 2011)

Results and Conclusions The study began in January 2017. Phase one is currently underway and its results, conclusions and recommendations will be presented at this conference.

\section{\begin{tabular}{|l|l}
\hline P-17 WE DON'T TALK ANYMORE - IMPROVING \\
\hline
\end{tabular} COMMUNICATION OF ADVANCE CARE PLANNING ON DISCHARGE FROM HOSPITAL}

${ }^{1}$ Lorna Fairbairn, ${ }^{2}$ Dee Traue. ${ }^{1}$ Sue Ryder Manorlands Hospice, West Yorkshire, UK; ${ }^{2}$ East and North Herts NHS Trust, UK

10.1136/bmjspcare-2017-hospice.44

Background Research shows that more than 30\% of hospital inpatients over 85 may die within the next year. Advance Care Planning (ACP) has been shown to increase achievement of preferred place of death and decrease unnecessary hospital admissions in the last year of life. Accordingly, local Elderly Care discharge summaries include a mandatory ACP section. As part of the 'Building on the Best' quality improvement programme, our Trust is focusing on improving handover of ACP information as people move between healthcare settings.

Aims To determine best practice in sharing information on ACP between hospital and community services

To review current transfer of information about ACP on discharge from an Elderly Care ward.

Method We reviewed relevant literature about transfer of information on discharge summaries and retrospectively audited 30 discharges from an elderly care ward. We recorded inclusion of key ACP topics, such as cardiopulmonary resuscitation status and preferred place of death, as well as deaths within the subsequent six months.

Results Literature review highlighted importance of high quality information in discharge summaries to decrease inappropriate readmission in last year of life. No discharge summaries audited included any information in the 'mandatory' ACP section

$50 \%$ documented DNAR status separately

$53 \%$ of patients were readmitted to our hospital within six months

$30 \%$ died within six months. 